Editorial

\title{
Acknowledgment of Reviewers of Challenges in 2020
}

\author{
Challenges Editorial Office
}

MDPI AG, St. Alban-Anlage 66, 4052 Basel, Switzerland

Peer review is the driving force of journal development, and reviewers are gatekeepers who ensure that Challenges maintains its standards for the high quality of its published papers. Thanks to the cooperation of our reviewers, in 2020, the median time to first decision was 19 days and the median time to publication was 38.5 days. The editors would like to express their sincere gratitude to the following reviewers for their precious time and dedication, regardless of whether the papers were finally published:

Adami, Renata

Ahmadinejad, Mitra

Amicucci, Antonella

Angeloni, Giulia

Ascencio-Vásquez, Julián

Badea, Dorel

Baum, Seth

Benaabidate, Lahcen

Benatar, Solly

Bettoni, Marco

Bharti, Vidhi

Bishoge, Obadia

Brabec, Jan

Bråtveit, Magne

Brown, Mark

Bryant, Christopher

Cai, Mengfan

Calevo, Jacopo

Carpenter, Alexander

Castro-Colin, Miguel

Cavallo, Aurora

Celli, Daniele

Chiang, Hsin-I

Cho, Sung-Hyoun

Demartini, Paola

Erhabor, Edobor Michael

Estellés, Marta

Fernández-Martínez, María Del Mar

Folmer, Britta

Francik, Slawomir

Frodeman, Robert

Gagliano, Monica

Ganzfried, Sam

Gentili, Denis

Giammarchi, Marco

Glibowski, Paweł

Glowacz, Adam

Gonçalves, Ângela

Guerrero, Francisco

Haluza, Daniela

Hele, Karl

Hernandez-Escobedo, Quetzalcoatl

Herrera, Miguel Angel

Huang, Hung-Pin
Ivanov, Peter

Jiménez-Fernández, Eduardo

Jose, Marco

Karbowski, Adam

Kim, Hye-Mi

Klöck, Carola

Kozlinska, Inna

Kurppa, Sirpa

Liu, Hailong

Lonsdale, Kate

Maghsoudi, Tahereh

MAK, Hugo Wai Leung

Matus, Patricia Isabel

Medina-Ramírez, Iliana

Mees, Heleen

Michel, Florent

Morkunas, Mangirdas

Naicker, Nisha

Najjar, Mohammad

Nam, Taewoo

Nunes, Ana Raquel

Onder, Mehmet

Paszkiel, Szczepan

Pisaturo, Giuseppe Roberto

Privitera, Donatella

Redvers, Nicole

Roman, Michał

Saldo, Jordi

Salvia, Rosanna

Sarfraz, Muddassar

Satarug, Soisungwan

Siddiqui, Nadia

Siroux, Monica

Sriwastva, Mukesh Kumar

Sudol, Miroslaw

Triviño-Tarradas, Paula

Uşak, Muhammet

Vinokurov, Sergey

Wałdykowski, Piotr

Wang, Chuanyi

Wang, Gai-Ge

Wartalska, Katarzyna

Webb, Darren

Wikman Svahn, Per 
Wilson, Amanda

$\mathrm{Wu}$, Wei

$\mathrm{Xu}$, Zhenzhen
Zakrzewska-Półtorak, Alicja

Zamora-Polo, Francisco 